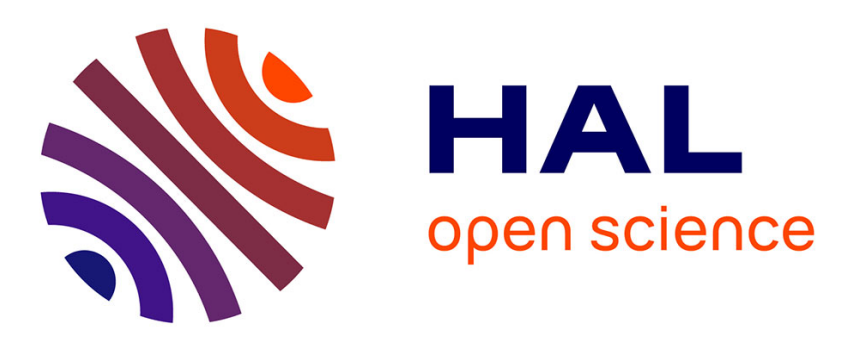

\title{
Emprendimiento en dos universidades públicas: universidad de Guanajuato, México y Université de Lorraine, France
}

María Guadalupe Perez-Zavala, Rubén Molina Sánchez, Christophe Schmitt, Alejandra López Salazar

\section{To cite this version:}

María Guadalupe Perez-Zavala, Rubén Molina Sánchez, Christophe Schmitt, Alejandra López Salazar. Emprendimiento en dos universidades públicas: universidad de Guanajuato, México y Université de Lorraine, France. Projectics / Proyéctica / Projectique, 2019, 2 (23), pp.99-116. 10.3917/proj.023.0099 . hal-02365464

\section{HAL Id: hal-02365464 \\ https://hal.univ-lorraine.fr/hal-02365464}

Submitted on 6 Dec 2020

HAL is a multi-disciplinary open access archive for the deposit and dissemination of scientific research documents, whether they are published or not. The documents may come from teaching and research institutions in France or abroad, or from public or private research centers.
L'archive ouverte pluridisciplinaire HAL, est destinée au dépôt et à la diffusion de documents scientifiques de niveau recherche, publiés ou non, émanant des établissements d'enseignement et de recherche français ou étrangers, des laboratoires publics ou privés. 


\section{EMPRENDIMIENTO \\ EN DOS UNIVERSIDADES PÚBLICAS: UNIVERSIDAD DE GUANAJUATO, MÉXICO Y UNIVERSITÉ DE LORRAINE, FRANCE}

\section{María Guadalupe Pérez-Zavala}

Universidad de Guanajuato

\section{Rubén Molina Sánchez}

Universidad de Guanajuato

\section{Christophe Schmitt}

Université de Lorraine

\section{Alejandra López Salazar}

\section{RESUMEN}

En la actualidad el espíritu emprendedor tiene el potencial de empoderar y transformar (Morris y Kuratko, 2014). Los ecosistemas emprendedores surgen como estrategia de desarrollo económico que se basa en la creación de apoyo al entorno y que fomentan nuevas empresas innovadoras (Spigel y Harrison, 2017). Por tal razón, universidades a nivel internacional han integrado ecosistemas de emprendimiento dentro de su organización; tal es el caso del ecosistema de Vinculación, Innovación, Desarrollo y Aplicación del conocimiento (VIDA) en la Universidad de Guanajuato, México y el Pôle Entrepreneuriat Etudiant De Lorraine (PEEL) en l'Université de Lorraine, France. Este documento hace un análisis sobre los datos de la Global University
Entrepreneurial

Spirit

Students' Survey (GUESSS) de algunas variables entre México y Francia; se utilizan estadísticos descriptivos, tablas de contingencia y estadísticos de prueba como $\mathrm{Chi}^{2}$ y Gamma. En cuanto a dichos ecosistemas en ambos países se hace un comparativo de lo que realizan. Se concluye que VIDA puede adoptar medidas que realiza el PEEL, como estrategia de mejora.

Palabras clave: Emprendimiento, universidad, ecosistema emprendedor, intenciones emprendedoras 


\section{ABSTRACT}

At present, entrepreneurship has the potential to empower and transform (Morris and Kuratko, 2014). Entrepreneurial ecosystems emerge as an economic development strategy based on creating support for the environment and fostering new innovative companies (Spigel and Harrison, 2017). For this reason, universities at the international level have integrated entrepreneurship ecosystems within their organization; such is the case of the Vinculation, Innovation, Development and

Application of Knowledge (VIDA) ecosystem at the University of Guanajuato, in Mexico and the Pôle Entrepreneuriat Étudiant de Lorraine (PEEL) at the Université de Lorraine, in France. This document analyzes the data of the Global
University Entrepreneurial Spirit Students' Survey (GUESSS) of some variables between Mexico and France; Descriptive statistics, contingency tables and test statistics such as Chi2 and Gamma are used. Regarding these ecosystems in both countries, a comparison of what they do is made. It is concluded that VIDA can adopt measures carried out by the PEEL, as an improvement strategy.

Keywords: Entrepreneurship, university, entrepreneurial ecosystem, entrepreneurial intentions

\section{RÉSUMÉ}

À l'heure actuelle, l'entrepreneuriat a le potentiel d'autonomiser et de transformer (Morris et Kuratko, 2014). Les écosystèmes entrepreneuriaux apparaissent comme une stratégie de développement économique fondée sur la création d'un soutien pour l'environnement et la promotion de nouvelles entreprises innovantes (Spigel et Harrison, 2017). Pour cette raison, les universités au niveau international ont intégré les écosystèmes d'entreprenariat au sein de leur organisation; Tel est le cas de l'écosystème VIDA IVinculation, Innovation, Développement et Application du savoir) de l'Université de Guanajuato (Mexique) et du Pôle Entrepreneuriat Étudiant de Lorraine (PEEL) de l'Université de Lorraine en France. Ce document analyse les données de l'enquête mondiale sur l'esprit d'entreprise des étudiants de l'Université (GUESSS) de certaines variables entre le Mexique et la France; Des statistiques descriptives, des tableaux de contingence et des statistiques de test telles que Chi2 et Gamma sont utilisés. En ce qui concerne ces écosystèmes dans les deux pays, une comparaison est faite de ce qu'ils font. Il est conclu que VIDA peut adopter les mesures mises en cuvre par le PEEL en tant que stratégie d'amélioration.

Mots-clés: Entrepreneuriat, université, écosystème entrepreneurial, intentions entrepreneuriales 


\section{INTRODUCCIÓN}

La universidad pública responde a una política de Estado, en la que se ofrece educación a la población; y constituye un pilar fundamental en la creación de identidad y referencias culturales para las naciones, por tanto, tanto en México como en Francia, las universidades publicas juegan papel relevante en la promoción del emprendimiento (Marín, Morúa, Rivera y Schmitt, 2013).

Como lo mencionan Herrera C. y Montoya L. (2013) el emprendimiento es un área del conocimiento nueva en el ámbito global de la investigación (Matiz, 2009); a partir de los años ochenta la creación de empresas comenzó a tomar un importante auge como campo de investigación científica (Stevenson y Jarillo, 1990; Bygrave y Hofer, 1991; Díaz, Urbano y Hernández, 2005). Para Marín et al. (2013) la globalización de los mercados ha marcado una creciente necesidad de competitividad; y el desarrollo económico de los países se ha ligado al espíritu de emprendimiento de sus pobladores.

Del papel de la universidad en el desarrollo del espíritu empresarial, se ha escrito relativamente poco, específicamente de cómo la universidad puede contribuir a través de la educación al apoyo empresarial y a las funciones de red y de ser emprendedor (Davey, Hannon y Penaluna, 2016). Sin embargo, para Marín et al. (2013) en las sociedades modernas, la universidad mantiene un rol importante en la formación y educación de la población; refiriéndose al fomento de la cultura emprendedora y a las capacidades ligadas al emprendimiento, como lo son: la concepción, manejo y comprensión de riegos, incertidumbres y del establecimiento de varios escenarios como base para la toma de decisiones.

También es imprescindible destacar que se deben evaluar los programas de emprendimiento con los que cuentan las universidades, aunado a que los educadores y/o formadores tengan una comprensión más completa de lo que desean lograr en dicho programa desde el inicio, a fin de asegurar una evaluación más precisa de los resultados (Henry, Hill y Leitch, 2005).

Para Morris y Kuratko (2014) el espíritu empresarial tiene el potencial de empoderar y transformar. La clave para la prosperidad individual y organizacional en un mundo dinámico, amenazador y complejo es la capacidad de pensar y actuar de una manera más emprendedora. La llamada ola mundial de desarrollo económico a esta nueva etapa de espíritu empresarial y de innovación. Mientras que, en el contexto de la educación, se puede argumentar que el estudiante en riesgo es el que no está preparado para esta edad empresarial. Todo estudiante tiene el potencial; pero la mayoría carece de los conocimientos, actitudes, habilidades y capacidades que definen la competencia empresarial.

La importancia de hacer un enfoque específico en las universidades, se basa en el modelo de ecosistema emprendedor que propone el Global Entrepreneurship Monitor (GEM) (Flores, 2017). El cual hace énfasis en seis dimensiones primordiales que el emprendedor requiere para desarrollarse: gobierno; inversionistas; redes de comunicación y redes sociales; familia y amigos; comunidades y universidades. 
Por tal motivo, el objetivo de este trabajo de investigación es hacer una comparación entre los ecosistemas emprendedores de dos universidades públicas que participaron en la GUESSS 2016-2017; la Universidad de Guanajuato con su ecosistema VIDA y la Universidad de Lorena con su ecosistema PEEL, ubicados en México y Francia, respectivamente. Con la finalidad de esclarecer que acciones o medidas correctivas de mejora podrían adoptar el uno del otro.

Para realizar el análisis comparativo se obtiene información de una base internacional que tiene datos estandarizados y comparables; en la cual participan los estudiantes universitarios pertenecientes a las carreras administrativas, con proyecto en puerta o trabajando en su emprendimiento lya sean estudiantes o egresados) de los países que se comparan. Para el caso de México, hacer una medición con otro país (Francia) cuya historia de emprendimiento tiene más años y desde luego pertenece a una economía con mejor competitividad, resulta de gran utilidad como punto de referencia que permite analizar y plantear aspectos de mejora.

El GEM por ejemplo ha hecho comparativos de ambos países con la finalidad de buscar modelos exitosos y eficientes que sirvan para ser replicados en otras universidades; también para medir las distancias entre países en cuanto a avance emprendedor se refiere; el en caso México-Francia, el primero aspira a estar entre las mejores economías del mundo para el 2050 (Molina, 2019). No es secreto para nadie que Francia es una de las economías más fuertes de la actualidad (Gestión, 2018), es indiscutible que éstos países se desarrollan en diferentes contextos y sufren diferentes carencias, por tal razón, México debe inspirarse en países desarrollados para lograr su propósito en los próximos años.

Aunado a lo interior, el equipo de trabajo que realiza esta investigación tiene fuerte interés en conocer a fondo dichos ecosistemas emprendedores universitarios para conocer las mejores prácticas de una configuración de acompañamiento de estudiantes desde el contexto universitario como es el que tiene el Instituto PEEL en la Universidad de la Lorena en Francia con sus estudiantes, para más adelante realizar una propuesta concreta de mejora a la Universidad de Guanajuato.

Como lo dijo Amorós (2018) para que una universidad tenga reconocimiento continuo y éxito en cualquiera de sus aspectos: académicos, deportivos, emprendedores, directivos, de docencia, etc. no le caería mal echar un vistazo a lo que están haciendo las mejores.

Este artículo se desarrolla en cuatro secciones: la primera hace referencia al emprendimiento como campo de investigación; en la segunda se encuentran diferentes definiciones de emprendedor; la tercera delimita los ecosistemas emprendedores (VIDA y PEEL) de dos universidades públicas: Universidad de Guanajuato y Universidad de Lorena; y finaliza con la cuarta sección donde se encuentran los resultados y conclusiones. 


\section{REVISIÓN DE LA LITERATURA}

\section{Emprendedor}

El término "emprendedor" proviene de la voz castellana "emprender" y que proviene del latín "tomar". Un emprendedor es la persona que emprende y se determina a hacer y ejecutar, con resolución o empeño algún proyecto considerable, arduo y de envergadura que requiera esfuerzo y dedicación" (Nuñez, 2013).

Para Cánovas, Konate y Rubio (2014) la palabra emprendedor, es un término de origen francés, que deriva de la palabra entrepreneur: se utiliza comúnmente para describir a la persona que organiza y opera una o varias empresas, asumiendo cierto riesgo financiero en el emprendimiento.

El término emprendedor fue definido por primera vez por el economista anglo-francés Richard Cantillon como la persona que paga un cierto precio para revender un producto a un precio incierto, por ende, tomando decisiones acerca de obtención y uso de recursos, admitiendo consecuentemente el riesgo en el emprendimiento (Díaz, 2016).

Por un lado Schumpeter (1934) dice que: “Los emprendedores son innovadores que buscan destruir el statu-quo de los productos y servicios existentes para crear nuevos productos y servicios". Mientras que Drucker menciona que: "un emprendedor busca el cambio, responde a él y explota sus oportunidades. La innovación es una herramienta específica de un emprendedor, por ende, el emprendedor efectivo convierte una fuente en un recurso" (Sánchez, 2005).

Como bien refieren Pierre-André y Molina (2012) a uno de los investigadores más antiguos del tema de emprendimiento, Cole, quien lo definía como una actividad que posibilita a crear, sostener y agrandar una empresa rentable. La OCDE en 2017 afirmó que el emprendimiento es una forma de ver las cosas y un proceso para crear y desarrollar actividades económicas con base en el riesgo, la creatividad y la innovación, al gestionar una organización nueva o existente.

Hoy en día la importancia del emprendedor es imprescindible, pero también es sustancial considerar el contexto en donde se desarrolla o forma uno de ellos, a lo que en los últimos años se ha convertido en boga de la investigación científica: ecosistemas emprendedores; para Napier y Hansen (2011), Malecki (2011) y Kantis y Federico (2012) el concepto de ecosistemas emprendedores es considerado como un enfoque emergente; sin embargo, el GEM nombra ecosistema emprendedor a seis dimensiones que marca como esenciales para que el emprendedor se desenvuelva (Flores, 2017).

\section{Ecosistema Emprendedor}

Para Spigel et al. (2017) los ecosistemas emprendedores son muy prometedores como marco conceptual para comprender las relaciones entre el proceso 
de emprendimiento y su entorno local; son una herramienta de política para ayudar a las regiones a catalizar una economía sostenible y dirigida por el espíritu emprendedor.

Para Flores (2017) las dimensiones que el GEM enlista para consolidar un ecosistema emprendedor ideal son: gobierno; inversionistas; redes de comunicación y redes sociales; familia y amigos; comunidades y universidades. Él expone que cada una de las dimensiones es importante para complementar el emprendimiento y que el emprendedor culmine eficaz y eficientemente su objetivo. Refiriéndose a cada una de ellas de la siguiente manera:

1. Gobierno. Como principal pilar del Estado, es la autoridad que dirige, controla y administra sus instituciones; por tal razón funge como eje primordial en la elaboración de leyes que apoyen a los emprendedores.

2. Inversionistas. Son todas aquellas personas que apuestan por la idea del emprendedor, confían en las proyecciones que éste presenta y deciden hacer una aportación económica para que comience el negocio y reditué lo antes posible.

3. Redes de comunicación y redes sociales. En los últimos años los contactos que los emprendedores pueden generar tanto personales como en línea, les puede traer grandes avances en el desarrollo de sus emprendimientos.

4. Familia y amigos. Son pilares importantes porque un emprendedor siempre está rodeado de ellos, son las personas en las que confían, más allegadas y a las que siempre preguntarán y pedirán apoyo.

5. Comunidades. Es importante reconocer las necesidades de la sociedad para poder ofrecer lo justo. Aunado a que en la sociedad cada vez se pueden detectar más emprendedores que inspiran a otros.

6. Universidades. La universidad, como formador de sus estudiantes, está integrando técnicas y estrategias que motiven e impulsen a sus alumnos a emprender.

Todas en conjunto hacen un ecosistema emprendedor idóneo para que un emprendedor pueda desarrollarse, sin embargo, eso no quiere decir que cada una de las dimensiones no pueda estar en constante mejora para poder complementar a las otras (Flores, 2017).

Según un reporte del GEM (2017) donde muestra los perfiles de los países en tema de condiciones de marco emprendedor; México y Francia en cuanto a educación emprendedora se refiere, se encuentran con una similitud de 3.5; lo que nos demuestra que aunque ambos difieran en algunas ámbitos como contexto, economía y desarrollo de país; tienen la similitud de que los universitarios (que compren de los 18 a los 30 años) quieren emprender en algún momento de su vida (intenciones); lo que resulta interesante analizar es el porcentaje de los que desean hacerlo inmediatamente después de egresar o pasados algunos años de haber concluido su carrera.

En las últimas cuatro décadas, el espíritu emprendedor ha crecido dentro de las universidades más rápido que prácticamente cualquier otra área 
de la búsqueda intelectual. Y parece que el ritmo se está acelerando con más universidades que buscan desarrollar programas y centros enfocados al emprendimiento. Sin embargo, la comprensión de cómo construir programas de emprendimiento que potencien y transformen sigue siendo un desafío para algunas instituciones (Morris et al., 2014).

Para Spigel et al. (2017) los ecosistemas emprendedores surgen como estrategia de desarrollo económico que se basa en la creación de apoyo al entorno y que fomentan nuevas empresas innovadoras. Representados por entornos culturales, sociales, económicos y políticos dentro de una región que apoyan el emprendimiento de alto crecimiento.

\section{Ecosistema emprendedor universitario}

La literatura sobre emprendimiento universitario se está expandiendo rápidamente, tanto en los Estados Unidos, Europa y alrededor del mundo (Rothaermel, Agung y Jiang, 2007). Es ampliamente aceptado que el sistema educativo de las universidades debe proporcionar un entorno académico que sirva de catalizador para las nuevas empresas de alta tecnología (Franke y Lüthje, 2004).

En su término más general y amplio, la universidad todavía se considera hoy como el centro más importante de cualquier sociedad en términos de investigación científica, transferencia de conocimiento y difusión de la cultura humanista. Sin embargo, en los últimos años, la universidad ha adquirido un papel cada vez más importante como foco estratégico de innovación, creatividad y emprendimiento, tanto en sociedades de países desarrollados como en regiones en desarrollo, donde es necesario impulsar el establecimiento de una red comercial que genera empleo y bienestar (Alonso-Gómez, MerigóLindahl, Peris-Ortiz, Rueda-Armengot y Rueda-Armengot, 2016).

Lima, Lopes, Nassif y Silva (2014) aseguran que las universidades y los colegios siempre pueden hacer más para brindar asistencia de calidad para el fomento de la capacidad emprendedora de sus estudiantes, la intención y la buena elección de carrera, en general. También afirman que lo ideal sería ofrecer un contexto experiencial a prueba donde los estudiantes puedan desarrollar sus ideas emprendedoras con el apoyo de los profesores, maestros, practicantes y otros.

Se han realizado varios estudios alrededor del mundo, donde se evalúa las actividades que se realizan en tema de emprendimiento (Alonso-Gómez et al., 2016), donde se incorporan iniciativas para estimular a los individuos a actuar de manera emprendedora (Klofsten, 2000) en donde se incluyen cátedras de emprendimiento dentro de las universidades, nuevos cursos y programas de capacitación; incluso hay empresas como Ericsson, Telia y Saab han establecido "unidades de innovación" para estimular nuevas ideas y utilizar la energía y las ideas de sus empleados.

También se observó la disposición de los estudiantes y el ambiente interno en universidades públicas cuando se implementa educación emprendedora. El resultado fue que los estudiantes demuestran disposición, en términos de 
habilidades y capacidades emprendedoras. Sin embargo, dentro del ambiente interno de las universidades públicas, la preparación para la educación emprendedora sigue siendo, desde la perspectiva de los estudiantes, imperfecta y requiere mejoras si estas instituciones respaldan la educación emprendedora futura (Othman, Hashim y Wahid, 2012).

Dichas investigaciones se toman como referencia en esta investigación para reafirmar que la dimensión de universidad dentro del ecosistema emprendedor completo es una parte fundamental; y que es necesario que investigadores se den a la tarea de buscar más a fondo qué es lo que se debe realizar y de qué manera para infundir el espíritu emprendedor en sus estudiantes y que sus proyectos sean exitosos.

\section{Ecosistema emprendedor universitario en la Universidad de Guanajuato (UG), México: Ecosistema de Vinculación, Innovación. Desarrollo y Aplicación del conocimiento de la Universidad de Guanajuato (VIDA UG)}

La Universidad de Guanajuato, ubicada en el ranking de las primeras 15 universidades en México, ocupa la posición número 13 (Saez, 2018), constituye el Ecosistema de Vinculación, Innovación, Desarrollo y Aplicación del conocimiento de la Universidad de Guanajuato (VIDA UG), para acercar la educación superior y sus capacidades académicas a la sociedad del estado de Guanajuato, y en concordancia con el reto de la Universidad del siglo XXI de transformar el conocimiento en desarrollo social, sustentable y económico (UG, 2018).

VIDA UG fue creado para propiciar la generación y la transferencia de conocimiento mediante productos específicos ajustados a los requerimientos del entorno. Cuenta con el soporte institucional de profesores, estudiantes y organismos de investigación, así como de redes académicas nacionales e internacionales dedicadas a la aplicación del conocimiento en el entorno social (UG, 2018).

La propuesta concibe a la innovación como un proceso amplio que debe abarcar y permear de manera transversal en todas las actividades de la institución como paso indispensable para transformarla en una universidad del futuro. Por tal razón, se enfoca en el potencial contenido y derivado de las capacidades científicas de la institución, sin limitarse al dominio tecnológico, sino abarcando con un espíritu integrador los rubros y las disciplinas sociales y humanísticas a efecto de generar un nuevo enfoque organizacional y una comunidad de innovación dentro de la institución (UG, 2018).

VIDA UG organiza talleres, pláticas y concursos en los cuatro campus de la Universidad de Guanajuato para que la "motivación emprendedora" llegue a más estudiantes. Imparte talleres de financiamiento, espíritu emprendedor, uso de redes sociales, propiedad intelectual, patentes, testimonio de 
emprendedores y empresarios exitosos; también realiza un concurso de creatividad e innovación anual (Pérez, 2019).

En cuestión de instalaciones, cuenta con un edificio ubicado en Silao a un costado del parque bicentenario, cuenta con varias aulas; sin embargo no está suficientemente equipado para cubrir las necesidades y requerimientos que los estudiantes demandan. Sumándole que el transporte para llegar al lugar escaso, ya que está muy alejado de la ciudad.

\section{Ecosistema emprendedor universitario en la Universidad de Lorena. Francia: Pôle Entrepreneuriat Étudiant De Lorraine (PEEL)}

La Universidad de Lorena (Université de Lorraine), ubicada en el ranking de las primeras 15 universidades en Francia, ocupa la posición número 11 (Metrics, 2018), constituye el Polo de Emprendimiento Estudiantil de Lorena (Pôle Entrepreneuriat Étudiant De Lorraine, PEEL) como parte de una convocatoria gubernamental para establecer polos de emprendimiento en el nivel superior. Fortalece el trabajo de las incubadoras, amplía la investigación sobre el emprendimiento y promociona la cultura emprendedora en toda la universidad (Marín et al., 2013).

El PEEL fue creado en 2011, tiene como objetivo desarrollar la cultura emprendedora dentro de la Universidad de Lorena. Lo que implica concientización, capacitación y apoyo. En 2017, con 194 estudiantes-empresarios, la Universidad de Lorena es la universidad más emprendedora de Francia por tercer año consecutivo (UL, 2017).

PEEL tiene un campus específico para que los estudiantes puedan desenvolver y desarrollar su habilidad emprendedora; en donde desde que entran se les hace una entrevista para conocer que es lo que quieren hacer y poder orientarlos, guiarlos y apoyarlos desde el inicio. Reciben talleres de distintos temas que les pueden servir para su emprendimiento: plan de negocio, financiamiento, asesorías personalizadas, seguimiento del proyecto, clases compartidas con otros emprendedores, convivencia y trabajo con otros emprendedores.

Sus instalaciones son de primera calidad, es un campus ubicado algo lejos del centro de la ciudad pero con fácil acceso por medio de transporte público. Sus puertas y aulas están abiertas de $8 \mathrm{am}$ a $6 \mathrm{pm}$ para que los estudiantes acudan siempre que lo necesitan; hay personal capacitado y especializado para resolver dudas y apoyar en todo momento.

\section{Método}

Esta investigación presenta una revisión de literatura y un análisis bajo un enfoque cuantitativo, de tipo exploratorio y descriptivo, que tiene como objetivo analizar los resultados arrojados por la GUESSS 2016 para México y Francia 
en cuanto a edad de los estudiantes universitarios y sus intenciones emprendedoras al término de su carrera y después de cinco años de haber concluido; después se hace un análisis sobre la información específica que se encuentra de la Universidad de Guanajuato, México y datos proporcionados en EtudiantsEntrepreneurs by PEEL por Schmitt (2016) y una entrevista en 2012 (Marín et al., 2013) para la Universidad de Lorena.

Este documento se desprende de una investigación más amplia en la cual se relacionan diferentes variables utilizadas por la GUESSS; sin embargo para ésta se toman en cuenta solo tres: edad, intenciones emprendedoras al terminar la carrera y después de cinco años. La edad es importante para demostrar que los jóvenes universitarios de entre 18 y 30 años son las personas más prometedoras para emprender y contribuir al desarrollo y crecimiento económico del país; ya que son el futuro (Gobierno de México, 2019), las intenciones al concluir la carrera y después de cinco años es importante conocerlas para saber porque los estudiantes están tomando esa decisión y poder renovar y reestructurar lo que se hace para mejorar.

De la base de datos internacional de la GUESSS (Sieger, 2017); se realizan estadísticos descriptivos sobre la información de los estudiantes de México y Francia; aunado a un análisis de tablas de contingencia considerando la edad como variable independiente y las intenciones de emprender de los universitarios terminado la carrera y después de cinco años de haber concluido, como variables dependientes, con objetivo de que quede justificado la importancia de crear o desarrollar ecosistemas emprendedores dentro de las universidad en respuesta a la solicitud de sus estudiantes, los cuales por su edad y etapa universitaria, demuestran un interés hacia el emprendimiento. También se realizaron estadísticos de prueba: Chi2 para demostrar si las variables son estadísticamente significativas; y Gamma para demostrar si existe una relación estadísticamente significativa entre las variables y Gamma para evaluar la fuerza de esa relación.

Los datos que se tomaron en cuenta para dicho análisis son las respuestas de los estudiantes que pertenecen a las universidades de Francia y México; y que participaron en la recolección de la GUESSS en su edición 2016-2017; y sus respuestas proporcionadas en cuanto a sus intenciones es mientras siguen siendo estudiantes; este instrumento internacional y estandarizado no les hace un seguimiento a dichos estudiantes ya cuando son egresados para saber que es lo que reamente hicieron.

\section{Resultados}

Los primeros resultados que se muestran a continuación son los descriptivos de ambos países: México y Francia; para las tres variables que se están considerando: intenciones inmediatamente terminando la carrera, intenciones después de cinco de terminar la carrera y edad.

El número de estudiantes universitarios que participaron para la GUESSS 2016 fue de 1,207 para el caso de México y de 714 para el caso de Francia. Los resultados fueron analizados con ayuda del software Statistical Package for the Social Sciences (SPSS). 
En la Tabla 1 se puede observar que tanto para México como para Francia, el mayor porcentaje de participación es para los universitarios menores de 24 años; aunque es más alta la participación para México con $89.0 \%$ que para Francia con $80.5 \%$. La participación más baja para ambos países es para universitarios mayores de 31 años.

\begin{tabular}{|l|c|c|}
\cline { 2 - 3 } \multicolumn{1}{c|}{} & \multicolumn{2}{c|}{ Edad } \\
\cline { 2 - 3 } \multicolumn{1}{c|}{} & MEX & FRA \\
\hline - de 24 años & 89.0 & 80.5 \\
\hline de 25 a 30 años & 9.3 & 15.9 \\
\hline + de 31 años & 1.7 & 3.6 \\
\hline
\end{tabular}

Fuente: Elaboración propia con base en datos de la GUESSS, 2016 para México y Francia.

Tabla 1. Participación en la GUESSS 2016; por categoría de edad y por país (\%)

En cuanto a las intenciones de los estudiantes se refiere, el procesamiento de los datos en el estadístico revela que casi el $90 \%$ de los universitarios mexicanos prefiere ser empleado inmediatamente después de terminar la carrera y un poco más del $80 \%$ también lo desea pasados cinco años del termino de la misma. Sin embargo para los universitarios franceses la intención de ser empleado inmediatamente después de terminar la carrera es menor al $50 \%$; pero al pasar cinco años de haber concluido su carrera, solo un $23 \%$ conserva la intención de ser empleado. Otro resultado destacable es que los universitarios que quieren ser emprendedores inmediatamente después de terminar su carrera, tanto como para México como para Francia, es muy bajo: $5 \%$ y $12 \%$ respectivamente; no obstante, para la variable de intenciones emprendedoras después de 5 años de haber terminado la carrera difieren significativamente, con poco más de $35 \%$ para los mexicanos y casi $66 \%$ para los franceses. En cuanto a ser sucesor de alguna empresa familiar, se ve claramente una ligera inclinación a que los estudiantes universitarios en Francia esperan heredar una empresa con un porcentaje más alto de lo que esperan los universitarios de México. Y finalmente, para los mexicanos es menos común responder que no saben que van a hacer después de terminar la carrera y cinco años más tarde (Véase Tabla 2).

\begin{tabular}{|l|c|c|c|c|}
\cline { 2 - 5 } \multicolumn{1}{c|}{} & \multicolumn{2}{c|}{$\begin{array}{c}\text { Intenciones inmediatamente } \\
\text { después de terminar la carrera }\end{array}$} & \multicolumn{2}{c|}{$\begin{array}{c}\text { Intenciones después de } 5 \text { años } \\
\text { de haber terminado la carrera }\end{array}$} \\
\cline { 2 - 5 } \multicolumn{1}{c|}{ EmPX } & FRA & MEX & FRA \\
\hline Empleado & 87.0 & 46.6 & 81.4 & 23.1 \\
\hline Sucesor & 5.0 & 36.1 & 12.1 & 65.9 \\
\hline Otro/aún no sé & 1.7 & 6.2 & 4.8 & 7.5 \\
\hline
\end{tabular}

Fuente: Elaboración propia con base en datos de la GUESSS, 2016.

Tabla 2. Intenciones de los alumnos; para ambos países: México y Francia (\%) 
Para analizar la relación de la edad con las intenciones de emprendimiento se realizó una tabla cruzada, en la cual se puede observar que la mayoría de los universitarios de cualquiera de ambos países, tiene preferencias de ser empleado sin importar el rango de edad al que pertenezca. En cambio de los que quieren ser emprendedores, para México en el rango de edad de 25-30 años, disminuye ligeramente de lo que había presentado para los menores de 24 años; pero para los mayores de 31 años aumenta notoriamente la intención; en cambio para Francia mientras más edad del universitario, más aumenta su intención de emprender (Véase Tabla 3).

\begin{tabular}{|l|c|c|r|r|r|c|c|c|}
\hline Intención & \multicolumn{2}{|c|}{ Empleado } & \multicolumn{2}{c|}{ Emprendedor } & \multicolumn{2}{c|}{ Sucesor } & \multicolumn{2}{c|}{ Otro / aún no sé } \\
\hline Edad & MEX & \multicolumn{1}{|c|}{ FRA } & MEX & \multicolumn{1}{c|}{ FRA } & MEX & FRA & MEX & FRA \\
\hline - de 24 años & 82.7 & 87.0 & 10.9 & 4.6 & 4.6 & 1.8 & 1.9 & 6.6 \\
\hline de 25 a 30 años & 70.5 & 85.6 & 9.7 & 6.3 & 4.1 & 0.9 & 1.7 & 7.2 \\
\hline + de 31 años & 80.0 & 84.0 & 20.0 & 12.0 & 0.0 & 4.0 & 0.0 & 0.0 \\
\hline
\end{tabular}

Fuente: Elaboración propia con base en datos de la GUESSS, 2016.

\section{Tabla 3. Relación entre edad y las intenciones inmediatamente después} de terminar la carrera $(\%)$

En la Tabla 4 se muestra la se muestra la prueba $\mathrm{Chi}^{2}$ para México y Francia en cuanto a las variables edad e intenciones de los universitarios inmediatamente después de terminar la carrera, encontrando que para el caso de México si existe una relación significativa entre la edad de los estudiantes y las intenciones de emprendimiento; mientras que para Francia, dicha relación no es estadísticamente significativa.

\begin{tabular}{|l|c|c|c|c|c|c|}
\cline { 2 - 7 } \multicolumn{1}{c|}{} & \multicolumn{3}{c|}{ MEX } & \multicolumn{3}{c|}{ FRA } \\
\cline { 2 - 7 } \multicolumn{1}{c|}{} & Valor & gl & $\begin{array}{c}\text { Sig. asintótica } \\
\text { (bilateral) }\end{array}$ & Valor & gl & $\begin{array}{c}\text { Sig. asintótica } \\
\text { (bilateral) }\end{array}$ \\
\hline Chi-cuadrado & 15.667 & 6 & .016 & 5.802 & 6 & .446 \\
\hline $\begin{array}{l}\text { Razón de } \\
\text { verosimilitudes }\end{array}$ & 15.228 & 6 & .019 & 6.677 & 6 & .352 \\
\hline $\begin{array}{l}\text { Asociación lineal } \\
\text { por lineal }\end{array}$ & 1.573 & 1 & .210 & .041 & 1 & .840 \\
\hline N de casos válidos & 1201 & & & 696 & & \\
\hline
\end{tabular}

Fuente: Elaboración propia con base en datos de la GUESSS, 2016.

Tabla 4. Prueba Chi2 para: edad e intenciones al terminar la carrera

Otra prueba estadística que se utilizó fue Gamma que sirve para evaluar la fuerza de la relación entre las variables; la cual demuestra nuevamente que para México, las variables edad e intenciones inmediatamente después de salir de la carrera si hay relación significativa; sin embargo la fuerza de dicha relación es muy débil (Véase Tabla 5). 


\begin{tabular}{|l|l|c|c|c|c|}
\cline { 3 - 6 } \multicolumn{2}{c|}{} & Valor & Error típ. asint. & T aproximada & Sig. aproximada \\
\hline \multirow{2}{*}{ México } & Gamma & .256 & .087 & 2.427 & .015 \\
\cline { 2 - 6 } & N de casos válidos & 1201 & & & \\
\hline \multirow{2}{*}{ Francia } & Gamma & .055 & .124 & .428 & .669 \\
\cline { 2 - 6 } & N de casos válidos & 696 & & & \\
\hline
\end{tabular}

Fuente: Elaboración propia con base en datos de la GUESSS, 2016.

Tabla 5. Prueba estadística: Gamma

A continuación se presenta una tabla comparativa entre VIDA y PEEL que son los ecosistemas de la Universidad de Guanajuato, México y de la Universidad de Lorena, Francia, las cuales también fueron partícipes de la GUESSS 2016.

\begin{tabular}{|c|c|c|}
\hline & Universidad de Guanajuato (UG) & Universidad de Lorena (UL) \\
\hline & VIDA (MEX) & PEEL (FRA) \\
\hline Objetivos & $\begin{array}{c}\text { Propiciar la generación y la } \\
\text { transferencia de conocimiento } \\
\text { mediante productos específicos } \\
\text { ajustados a los requerimientos } \\
\text { del entorno. Potencializar la } \\
\text { estructura de la UG, fundamentar } \\
\text { en la responsabilidad social } \\
\text { universitaria, actuar con } \\
\text { sensibilidad para detectar } \\
\text { necesidades del entorno, asumir } \\
\text { una cultura de la innovación } \\
\text { y facilitar procesos para la } \\
\text { materialización de las soluciones. }\end{array}$ & $\begin{array}{l}\text { Desarrollar una cultura } \\
\text { del emprendimiento en } \\
\text { los estudiantes de la UL } \\
\text { y favorecer el desarrollo } \\
\text { territorial a través de la } \\
\text { iniciativa emprendedora. }\end{array}$ \\
\hline Slogan & Talento UG con la sociedad & Red que conecta \\
\hline Enfoque & $\begin{array}{l}\text { Integrar las iniciativas de } \\
\text { estudiantes y profesores en } \\
\text { proyectos multidisciplinarios } \\
\text { como corresponsables }\end{array}$ & $\begin{array}{c}\text { Proyectos internos de la } \\
\text { universidad }\end{array}$ \\
\hline $\begin{array}{l}\text { Política de } \\
\text { origen }\end{array}$ & $\begin{array}{c}\text { Plan Nacional de Desarrollo y } \\
\text { Ley orgánica de la UG }\end{array}$ & Política educativa \\
\hline Director & $\begin{array}{l}\text { Dr. en sistemas eléctricos, } \\
\text { investigador de la universidad }\end{array}$ & $\begin{array}{l}\text { Dr. en ciencias, investigador } \\
\text { de la universidad }\end{array}$ \\
\hline $\begin{array}{l}\text { Principios } \\
\text { de base }\end{array}$ & $\begin{array}{l}\text { Concibe a la innovación como un } \\
\text { proceso amplio que debe abarcar } \\
\text { y permear de manera transversal } \\
\text { en todas las actividades de } \\
\text { la institución como paso } \\
\text { indispensable para transformarla } \\
\text { una universidad del futuro. }\end{array}$ & $\begin{array}{c}\text { El emprendimiento supera la } \\
\text { creación de empresas para } \\
\text { ser una actitud de innovación. } \\
\text { Hay tres ejes: sensibilizar, } \\
\text { formar y acompañar. El } \\
\text { modelo se basa en la } \\
\text { creación de una unidad de } \\
\text { negocio. }\end{array}$ \\
\hline Resultados & No hay registro & 115 proyectos (Schmitt, 2016) \\
\hline Estudiantes & No hay registro & 136 (Schmitt, 2016) \\
\hline
\end{tabular}




\begin{tabular}{|l|c|c|}
\hline Instalaciones & Inadecuadas & Adecuadas y óptimas \\
\hline $\begin{array}{l}\text { Cursos } \\
\text { Talleres }\end{array}$ & $\mathrm{Si}$ & $\mathrm{Si}$ \\
\hline $\begin{array}{l}\text { Asesorías } \\
\text { personalizadas }\end{array}$ & No & $\mathrm{Si}$ \\
\hline $\begin{array}{l}\text { Entrevista de } \\
\text { ingreso }\end{array}$ & No & $\mathrm{Si}$ \\
\hline $\begin{array}{l}\text { Seguimiento } \\
\text { de proyectos }\end{array}$ & No & $\mathrm{Si}$ \\
\hline $\begin{array}{l}\text { Publicación y } \\
\text { difusión de lo } \\
\text { que se hace }\end{array}$ & No & $\mathrm{Si}$ \\
\hline
\end{tabular}

Fuente: Elaboración propia con base Schmitt (2016), UG (2018), Marín et al. (2013) y Pérez (2019).

\section{Tabla 6. Comparativo entre VIDA y PEEL}

\section{CONCLUSIONES}

Se hizo un análisis sobre los datos de la Global University Entrepreneurial Spirit Students' Survey (GUESSS) de las variables: edad, intenciones de los universitarios al terminar su carrera e intenciones de los universitarios después de cinco años de terminar su carrera entre México y Francia; se mostraron estadísticos descriptivos, una tabla de contingencia que muestra la asociación que tiene la variable edad con las intenciones que los estudiantes tienen al terminar sus estudios universitarios. También se realizaron estadísticos de prueba como: Chi $^{2}$ y Gamma.

La importancia de impulsar a que los jóvenes sean emprendedores aumenta cada día más; señalan Urbano, Díaz y Hernández (2007); sin embargo, las conclusiones más relevantes que se pueden rescatar después del análisis de los resultados, es que a México le queda mucho por hacer en materia de emprendimiento universitario; ya que 9 de cada 10 estudiantes tiene la intención de ser empleado en cuanto terminen su carrera profesional y 8 de cada 10 también lo preferirán después de cinco años de terminar su carrera; lo que es preocupante porque como ya fue mencionado en el documento la creación de empresas es fundamental para el desarrollo económico del país y el aumento de empleo, por lo tanto hay que tomar seriedad y darle la importancia necesaria a la parte de motivar e impulsar a los estudiantes a que desarrollen su espíritu emprendedor.

Otro resultado destacable es que las intenciones que tienen los universitarios para emprender algún negocio, ya sea al terminar sus estudios profesionales o bien, cinco años después es muy baja para ambos países. Lo que quiere decir que en promedio 1 de cada 10 lo quiere hacer inmediatamente después de graduarse; y pasados cinco años del termino de sus estudios profesionales en México 3 de cada 10 lo quieren hacer y en Francia 6 de cada 10. Conforme a lo que dijeron Urbano, et al. (2007, p.183) "es indiscutible en la actualidad la importancia que tiene la creación de empresas como motor de 
desarrollo de las economías modernas tanto en lo referente a la generación de ocupación como para el crecimiento económico"; los resultados anteriores no avalan que se esté impulsando y motivando al estudiante universitario a ser emprendedor y a que dirija su enfoque hacia la creación de empresas.

Hoy en día, hay un número cada vez mayor de universidades que incorporan programas de emprendimiento en sus universidades (Jansen, Zande, Brinkkemper, Stam y Varma, 2015); Siegel y Wright, 2015). Las iniciativas de educación empresarial pueden emplear el enfoque de administración de pequeñas empresas relativamente tradicionales así como también un enfoque más reciente de creación de empresas de alto crecimiento (Rideout y Gray, 2013).

En resumen, algunos programas se centran exclusivamente en las etapas de formación de jóvenes emprendedores a través de clases tradicionales y simples desafíos de creación de start-ups. Y otros programas establecen una red de actores con roles específicos y claros, cuyo objetivo compartido es formar, crecer y fortalecer emprendedores de diferentes niveles y en diferentes etapas (Siegel et al., 2015). A estos programas desarrollados en diferentes universidades alrededor del mundo, bien se les podría llamar ecosistemas emprendedores; sin embargo no hay un modo unánime de forma de evaluación de los resultados de dichos programas.

En cuanto a los ecosistemas universitarios en ambos países se refiere, del comparativo se deduce que VIDA lecosistema de la Universidad de Guanajuato, México), aunque tiene menos tiempo en funcionamiento que el PEEL (ecosistema de la Universidad de Lorena, Francial no revela resultados significativos de lo que propone como objetivos en su presentación. Lo que quiere decir que se pueden adoptar medidas realizadas en el PEEL, tales como: tener un registro de los proyectos que se inscriben, dar seguimiento a los emprendimientos de los estudiantes, llevar control de los que siguen en marcha, etc. todo lo anterior, con la finalidad de crear una estrategia de mejora para VIDA.

Se propone que VIDA pueda ofrecer una estructura más fuerte de su ecosistema universitario emprendedor, en donde, al igual que el PEEL pueda brindar en su cartera de servicios: acompañamiento, seguimiento, capacitaciones, parques industriales universitarios, difusión del programa emprendedor.

Recordemos que se deben tener presentes las 6 dimensiones que enlista el GEM como parte de los ecosistemas emprendedores son: 1) gobierno; 2) inversionistas; 3) redes de comunicación y redes sociales; 4) familia y amigos; 5) comunidades y 6) universidades. Para que un emprendedor pueda desenvolverse en excelentes condiciones, cada una de las 6 dimensiones tendría que estar funcionando correctamente e intentar mejorar continuamente, sin embargo esta investigación se enfoca únicamente en la sexta: universidades.

Lo anterior se menciona con la intención de enmarcar que el ecosistema universitario emprendedor no es la única dimensión que completa el ecosistema; puesto que una sola organización sería insuficiente para suministrar todo lo que requiere un emprendedor; sin embargo, se habla de un entorno de mejora en el cual la universidad es importante y se reafirma que es un agente dentro de un ambiente más amplio. Aunado a que como parte de las investigaciones del equipo de trabajo es fundamental proponer mejoras continuas a sus propios lugares de trabajo. 


\section{Limitaciones}

Las base de datos utilizada para el análisis de este documento, es la que tiene la información más actualizada hasta la fecha, GUESSS 2016-2017; sin embargo en el segundo semestre de 2018 y parte del primero de 2019 se estará ejecutando y recopilando información nuevamente, para mantener actualizada la base de datos internacional.

Cabe mencionar también, que esta investigación pertenece a una más grande (tesis de maestría) donde se consideran más variables medidas por el GUESSS e incluso dimensiones y categorías de un investigación cualitativa que se realizó a la par; pero por cuestiones de tiempo y espacio, se ha acotado a solo medir algunas en este documento.

\section{Direcciones y oportunidades futuras}

Hacer un nuevo análisis con la base de datos GUESSS 2018-2019 y una comparación de la evolución o retroceso que se haya tenido en esta brecha de 2 años en cuanto a la dimensión de universidad que pertenece al ecosistema emprendedor planteado por el GEM; específicamente de México y Francia.

\section{BIBLIOGRAFÍA}

Alonso-Gómez, J., Merigó-Lindahl, J. M., Peris-Ortiz M. \& Rueda-Armengot, C. (2016). Entrepreneurial Universities: Exploring the Academic and Innovative Dimensions of Entrepreneurship in Higher Education. Springer. Recuperado: https://books.google.com.mx/books?hl=es\&lr=\&id=2 d04DQAAQBAJ\&oi=fnd\&pg=PR5\&dq=entrepreneurial+universities+exploring+the+academic $+a$ nd+innovate+dimensions+of+entrepreneurship+in+higher+education \&ots=bhq5xkvn7K\&sig=9c WkTlo-_dljwbcbR93vuFSod3k\#v=onepage\&q=entrepreneurial\%20universities $\% 20$ exploring $\% 20$ the $\% 20$ academic $\% 20$ and $\% 20$ innovate $\% 20$ dimensions $\% 20$ of $\% 20$ entrepreneurship $\% 20$ in $\% 20$ higher\%20education\&f=false

Amorós, J. E. (comunicación personal, 22 de febrero, 2018).

Bygrave, W. \& Hofer, C. (1991). Theorizing about entrepreneurship, Entrepreneurship Theory and Practice, 13-22.

Cánovas, V., Konate, F. \& Rubio, C. (2014). Cultura emprendedora, iniciativa y espíritu empresarial. Mi Periódico Digital. Recuperado de: http://www.miperiodicodigital.com/2014/edicion2014/ palabras/25709cultura-emprendedora-iniciativa-espiritu-empresarial.html

Davey, T., Hannon, P. \& Penaluna, A. (2016). Entrepreneurship education and the role of universities in entrepreneurship: Introduction to the special issue. Industry and Higher Education. 30(3), 171-182. DOI: 10.1177/0950422216656699

Díaz, J., Urbano, D. \& Hernández, R. (2005). Teoría económica institucional y creación de empresas. Investigaciones Europeas de Dirección y Economía de la Empresa, 11(3), 209-230.

Díaz, M. (2016). El término emprendedor. Mi Periódico Digital. Recuperado de: http://www. miperiodicodigital.com/2016/grupos/lamentirabg-122/el-termino-emprendedor-598.html

Flores, M. A. (2017). Una mirada sistémica del emprendimiento y la innovación. Presentación en congreso CillEMIPYME 2017. Tecnológico de Monterrey.

Franke, N. \& Lüthje, C. (2004). Entrepreneurial intentions of business students - A benchmarking study. International Journal of Innovation and Technology Management, 1(03), 269-288.

Gestión. (2018). Las economías más grandes del mundo en 2018. Gestión. Recuperado de: https:// gestion.pe/economia/economias-grandes-mundo-2018-232078 
Global Entrepreneurship Monitor. (GEM, 2017). Economy Profiles. France. México. Recuperado de: https://www.gemconsortium.org/country-profiles

Henry, C., Hill, F. \& Leitch, C. (2005). Entrepreneurship education and training: can entrepreneurship be taught? Part I. Emeraldinsight, 47(2): 98-111. DOI: 10.1108/00400910510586524

Herrera, C. E. \& Montoya, L. A. (2013). El emprendedor: una aproximación a su definición y caracterización. The entrepreneur: An approach to its definition and characterization. Dialnet, 4(7), 10-31. Recuperado de: file:///C:/Users/Lu/Downloads/Dialnet-ElEmprendedor-4776922\%20 (1).pdf

Jansen, S., Zande, T., Brinkkemper, S., Stam, E. \& Varma, V. (2015). How education, stimulation, and incubation encourage student entrepreneurship: Observations from MIT, IIIT, and Utrecht University. The International Journal of Management Education, 13(2), 170-181.

Kantis, H. \& Frederico, J. (2011). Entrepreneurial Ecosystems in Latin America: the role of policies. Recuperado de: http://www.innovacion.gob.cl/wp-content/uploads/2012/06/EntrepreneurialEcosystems-in-Latin-America_the-role-of-policies.pdf

Klofsten, M. (2000). Training entrepreneurship at universities: a Swedish case. Journal of European Industrial Training, 24(6), 337-344.

Lima, E., Lopes, R. M., Nassif, V. \& Silva, D. (abril, 2014). Opportunities to Improve Entrepreneurship Education: Contributions Considering Brazilian Challenges. Journal of Small Business Management. Vol. 53, Issue 4.

Malecki, E. J. (2011). Connecting local entrepreneurial ecosystems to global innovation networks: open innovation, double networks and knowledge integration. International Journal of Entrepreneurship and Innovation Management, 14, 36-59.

Marín, A., Morúa, J., Rivera, I. \& Schmitt, C. (2013). Modelos de acompañamiento a emprendedores en México y Francia. En M. Marcelino, I. Rivera y M. Aguilar (Eds.), Desarrollo tecnológico y empresarial. Agentes y aplicaciones (197-224). Ciudad de México, México: Porrúa.

Matiz, F. (2009, mayo-agosto). Investigación en emprendimiento, un reto para la construcción de conocimiento. Revista EAN, 66, 169-182.

Metrics. (2018). Ranking Web de Universidades, Europa: Francia. Recuperado de: http://www. webometrics.info/es/Europe/France\%20

Molina, R. (comunicación personal, 16 de enero, 2019).

Morris, M. H. \& Kuratko, D. F. (2014), Building University 21st Century Entrepreneurship Programs that Empower and Transform, in Sherry Hoskinson, Donald F. Kuratko (ed.) Innovative Pathways for University Entrepreneurship in the 21st Century (Advances in the Study of Entrepreneurship, Innovation \&amp; Economic Growth, Volume 24) Emerald Group Publishing Limited, 1 - 24.

Napier, G. \& Hansen, C. (2011). Ecosystems for Young Scaleable Firms, FORA Group.

Nuñez, E. (2013). Las definiciones de Emprendedor y Empresario (Mensaje en un blog). Emprende y Prospera. Recuperado de: http://www.emprendeyprospera.com/las-definiciones-deemprendedor-y-empresario/\#.VVby9fmqoSU

Othman, N., Hashim, N. \& Wahid, H., (2012). Readiness towards entrepreneurship education: Students and Malaysian universities. Education + Training. Vol. 54. Issue: 8/9, pp. 697-708. Recuperado de: https://doi.org/10.1108/00400911211274837

Pérez, M. G. (2019). El ecosistema emprendedor universitario como indicador de impulso y motivación para que los estudiantes emprendan: el caso de la Universidad de Guanajuato. Tesis de maestría no publicada. Universidad de Guanajuato, Guanajuato, México.

Pierre-André, J. \& Molina, R. (2012). Una teoría sobre el emprendimiento regional en la economía del conocimiento: Una metáfora de las telenovelas policiacas. México, DF., México: Pearson Educacion.

Rideout, E. C., \& Gray, D. 0. (2013). Does entrepreneurship education really work? A review and methodological critique of the empirical literature on the effects of university-based entrepreneurship education. Journal of Small Business Management, 51(3), 329-351.

Rothaermel, F. T., Agung, S. D. \& Jiang, L. (2007). University entrepreneurship: a taxonomy of the literature. Industrial and corporate change, 16(4), 691-791.

Saez, C. (2018). Ránking de Universidades de México 2018. AméricaEconomía Intelligence. Recuperado de: https://mba.americaeconomia.com/articulos/notas/ranking-de-universidadesde-mexico-2018 
Sánchez, A. J. (2005). Peter Drucker, innovador maestro de la administración de empresas. Facultad de Ciencias Económicas y Administrativas, 69-89. Recuperado de: https://www.researchgate.net/ publication/265149719_PETER_DRUCKER_INNOVADOR_MAESTRO_DE_LA_ADMINISTRACION_ DE_EMPRESAS

Schmitt, C. (2016). Etudiants-Entrepreneurs by PEEL. Quelques chiffres. Manuscrito no publicado, Université de Lorraine, Nancy, La France.

Schumpeter, J. (1934). The Theory of Economic Development, Harvard University Press, Cambridge, Massachusetts.

Siegel, D. S. \& Wright, M. (2015). Academic entrepreneurship: time for a rethink?. British Journal of Management, 26(4), 582-595.

Sieger, P. (2017). GUESSS CEO. Base de Datos Internacional de la GUESSS 2016.

Spigel, B. \& Harrison, R. (2017). Toward a process theory of entrepreneurial ecosystems. Strategic Entrepreneurship Journal, 12(1), 151-168. DOI:10.1002/sej.1268

Stevenson, H. H. \& Jarillo, J. C. (1990). A Paradigm of Entrepreneurship: Entrepreneurial Management. Strategic Management Journal, 11, 17-27.

Universidad de Guanajuato. (UG, 2018). Ecosistema de Vinculación, Innovación, Desarrollo y Aplicación del conocimiento de la Universidad de Guanajuato (VIDA UG). Presentación. Recuperado de: http://www.ugto.mx/vidaug/que-es-vida-ug/presentacion

Université de Lorraine. (UL, 2017). Pôle Entrepreneuriat Étudiant De Lorraine (PEEL). Qui sommesnous? Recuperado de: http://peel.univ-lorraine.fr/pole-entrepreneuriat-etudiant-de-lorraine/ qui-sommes-nous/

Urbano, D., Díaz, J. C. \& Hernández, R. (2007). Evolución y principios de la teoría económica institucional. Una propuesta de aplicación para el análisis de los factores condicionantes de la creación de empresas. Investigaciones Europeas de Dirección y Economía de la Empresa, 13 (3), 183-198. Recuperado de http://www.redalyc.org/comocitar.oa?id=274120871010

María Guadalupe PÉREZ-ZAVALA. Maestra en Estudios Empresariales en la Universidad de Guanajuato Campus Celaya-Salvatierra. Emprendimiento Universitario. Jóvenes emprendedores.

Correo: mg.perezzavaladugto.mx

Rubén MOLINA SÁNCHEZ. Profesor-Investigador de Tiempo Completo en la Universidad de Guanajuato Campus Celaya-Salvatierra. Innovación. Emprendimiento. Crecimiento de la PyME.

Correo: humanarubendagmail.com

Christophe SCHMITT. Profesor-Investigador de Tiempo Completo en la Université de Lorraine. Cultura empresarial estudiantil. IDéO@. Espíritu empresarial. Acción emprendedora.

Correo: christophe.schmitt@univ-lorraine.fr

Alejandra LÓPEZ SALAZAR. Profesora-Investigadora de Tiempo Completo en la Universidad de Guanajuato Campus Celaya-Salvatierra. Capacidades tecnológicas. Estrategia empresarial.

Correo: alelopez.salazardyahoo.com 